\title{
Serum Bone Gamma Carboxyglutamic Acid-containing Protein in Primary Hyperparathyroidism and in Malignant Hypercalcemia

\author{
Comparison with Bone Histomorphometry
}

\author{
P. D. Delmas, B. Demiaux, L. Malaval, M. C. Chapuy, C. Edouard, and P. J. Meunier \\ Clinique de Rhumatologie and Institut Nationale de la Santé et de la Recherche \\ Medicale U. 234, Hôpital Edouard Herriot, Lyon, France
}

\begin{abstract}
Serum bone $\boldsymbol{\gamma}$-carboxyglutamic acid-containing (Gla) protein (sBGP), a sensitive and specific marker of bone turnover, was measured in $\mathbf{2 5}$ patients with primary hyperparathyroidism and in 24 patients with bone metastases with or without hypercalcemia. Despite similar levels of hypercalcemia, sBGP was increased in primary hyperparathyroidism $(14.2 \pm 9.6 \mathrm{ng} / \mathrm{ml}, P$ $<0.001)$, was decreased in malignant hypercalcemia $(3.1 \pm 2.8$ $\mathrm{ng} / \mathrm{ml}, \boldsymbol{P}<0.001$ ), and was normal in patients with bone metastases without hypercalcemia $(6.6 \pm 2.7 \mathrm{ng} / \mathrm{ml})$. In primary hyperparathyroidism, sBGP was correlated with serum immunoreactive parathyroid hormone $(r=0.90)$, calcium $(r=0.73)$, and with the adenoma weight $(r=0.79)$. After parathyroidectomy, sBGP slowly returned to normal values within 2-6 mo, suggesting that SBGP reflects increased bone turnover rather than a direct effect of parathyroid hormone on BGP synthesis at the cell level. An iliac crest biopsy was performed in 11 patients with primary hyperparathyroidism and in 9 cancer patients in a noninvaded area. SBGP was significantly correlated with all parameters reflecting bone formation but not with bone resorption. Patients with bone metastases were analyzed according to the presence or the absence of hypercalcemia. In contrast to normocalcemic patients who had normal sBGP, hypercalcemic patients had decreased SBGP $(P<0.001)$ and a lower bone formation at the cellular level $(P<0.05)$. Thus, biochemical and histological data suggest that an unknown humoral factor might be responsible for this uncoupling between increased resorption and decreased formation. This uncoupling, rather than local release of calcium by the metastatic process, might be responsible for hypercalcemia in patients with bone metastases.
\end{abstract}

\section{Introduction}

Primary hyperparathyroidism and cancer are the most frequent causes of hypercalcemia. In patients with primary hyperparathyroidism, increased bone turnover is a common finding. Iliac crest biopsy analysis shows an increase of both osteoclastic bone resorption and osteoblastic bone formation (1-6) and imbalance between both plays a role in the mechanism of hypercalcemia. In patients with malignant hypercalcemia related to solid tumors,

Address correspondence to Dr. Delmas, Hôpital Edouard Herriot, Pavillon E, Place d'Arsonval, 69374 Lyon, France.

Received for publication 10 December 1984 and in revised form 14 November 1985.

J. Clin. Invest.

(C) The American Society for Clinical Investigation, Inc.

0021-9738/86/03/0985/07 \$1.00

Volume 77, March 1986, 985-991 two mechanisms are commonly implicated according to the presence or the absence of bone metastases $(7,8)$. In the absence of bone metastases, hypercalcemia occurs as the result of secretion by the tumor of humoral calcemic factors. These putative factors have been shown to induce a marked uncoupling between increased bone resorption and decreased bone formation, which accounts for bone loss (9). Among patients with cancer-associated hypercalcemia, those with bone metastases constitute the largest group but they have received little attention in the literature. Hypercalcemia is usually attributed to local release of skeletal calcium caused by the metastatic process $(8,10)$, but the possible role of humoral factors has been suggested even in the presence of bone metastases (7). Increased osteoclastic bone resorption is a common finding $(11,12)$, but the level of bone formation has not been investigated systematically. Assessment of total bone turnover by noninvasive methods is crucial in order to answer this question, but available markers are insufficient. Serum alkaline phosphatase (sAP) ${ }^{1}$, an index of bone formation, and urinary hydroxyproline (uOH-Prol), an index of bone resorption, are not specific for bone tissue. They may be increased in situations with no abnormality of bone.

We have studied this problem by measuring serum bone $\gamma$-carboxyglutamic acid-containing (Gla) protein (sBGP) ${ }^{1}$, also called osteocalcin $(13,14)$. BGP is the most abundant noncollagenous protein of bone and it is specific for skeletal tissue. It is synthesized by bone cells and circulates in blood $(15,16)$. We lack accurate estimates of the fraction which is incorporated into bone and of the fraction released into the circulation, but sBGP has been found to be a sensitive indicator of bone turnover in patients with a variety of metabolic bone diseases (17-19), including primary hyperparathyroidism $(17,20)$. Using a sensitive radioimmunoassay (21), one of us has previously shown that sBGP is increased in patients with primary hyperparathyroidism and decreased in patients with hypoparathyroidism (22). Recently, we have shown that $\mathrm{SBGP}$ is a specific marker of bone formation but not resorption in patients with postmenopausal osteoporosis (23), a finding recently confirmed by others in patients with renal osteodystrophy (24).

In a prospective study, we have measured sBGP in a group of 25 patients with primary hyperparathyroidism before and after surgery and in 9 patients with hypercalcemia related to bone metastases. sBGP values were correlated with other markers of bone turnover (sAP and uOH-Prol) and with markers of parathyroid hormone secretion. Biochemical measurements were compared with histological measurement of bone turnover ob-

1. Abbreviations used in this paper: BGP, bone $\gamma$-carboxyglutamic acidcontaining (Gla) protein; PTH, parathyroid hormone; sAP, serum alkaline phosphatase; sBGP, serum BGP; sCa, serum calcium; sP, serum phosphate; sPTH, serum PTH; uCa, urinary calcium; uOH-Prol, urinary hydroxyproline. 
tained from iliac crest biopsies. Data were also compared with those obtained in 15 patients having bone metastases without hypercalcemia.

\section{Methods}

Subjects. We studied 25 consecutive patients ( 21 women, 55 \pm 11 -yr-old; 4 men, 55 \pm 12 -yr-old) admitted to the Rheumatology and Metabolic Bone Disease Unit for primary hyperparathyroidism. In 21 of them the diagnosis was confirmed by surgical removal of a parathyroid adenoma. In the remaining four the diagnosis was based on clinical and laboratory criteria including hypercalcemia and an elevated level of serum immunoreactive parathyroid hormone (sPTH). 18 patients had asymptomatic hypercalcemia, 5 had a history of urolithiasis, one had vertebral compression fractures, and one patient had severe hypercalcemia. Renal function was normal in all patients except one who had a moderate increase of serum creatinine (148 $\mu \mathrm{mol} / \mathrm{liter}$, normal $<110 \mu \mathrm{mol} / \mathrm{liter})$.

We also studied a group of nine patients ( $51 \pm 8$-yr-old) with hypercalcemia related to bone metastases. These were consecutive patients admitted to the Rheumatology and Metabolic Bone Disease Unit for bone metastases and hypercalcemia. Clinical features are indicated in Table I. sBGP was measured before therapy of hypercalcemia and primary tumor was discovered at the time of hypercalcemia. Therefore none of the patients had previously received chemotherapy.

A control cancer group comprised of patients with bone metastases without hypercalcemia was studied. They were 15 women $(50 \pm 13-y r-$ old) with breast cancer that were admitted to the Oncology Unit (Dr. M. Clavel, Centre Léon Berard, Lyon, France). X-ray examination showed that bone metastases were of the lytic type in six cases and of the mixed type in two cases. Bone metastases were detected only by bone scan in five cases and by bone biopsy in two cases. The number of bone metastases per patient was similar in the group of patients with hypercalcemia and in the group of patients without hypercalcemia ( $5 \pm 4$ and $5 \pm 5$, respectively, NS). Thirteen patients were being treated at the time of the study (Tamoxifen in 11 patients, ovariectomy in two cases, adrenalectomy in one case, two to three courses of chemotherapy in three cases).

Biochemistry. We obtained fasting morning blood samples for determinations of serum concentrations of calcium $(\mathrm{sCa})$, phosphate $(\mathrm{sP})$, sAP, BGP, and PTH. 24-h urine collection was obtained during two to three consecutive days for measurement of calcium $(\mathrm{uCa})$, total $\mathrm{uOH}$ Prol, and in some cases for cyclic AMP (cAMP). sBGP was measured with an assay similar to the radioimmunoassay described by Price and Nishimoto (16) with a modification of previously published methods (21). This assay uses antiserum (R $102 \mathrm{M})$ directed against bovine BGP and bovine BGP as a standard and tracer. After a 4-h incubation at $37^{\circ} \mathrm{C}$, antibody-bound and free ${ }^{125} \mathrm{I}$-labeled BGP are separated by the double-antibody method using a complex of sheep anti-rabbit IgG and polyethylene glycol (precipitating reagent; Cie. ORIS Industrie, Bagnols, France). The sensitivity of the assay is $0.05 \mathrm{ng}$ of bovine BGP per tube and all values for standards and samples were determined in triplicate. Dilution curves of serum samples from controls and from patients with either primary hyperparathyroidism or malignant hypercalcemia were found to be parallel. Control values were obtained in 107 age-matched normal women $(60 \pm 19$-yr-old, $\mathrm{sBGP}=6.4 \pm 2.2 \mathrm{ng} / \mathrm{ml})$ and in 23 agematched normal men $(53 \pm 9$-yr-old, sBGP $=5.7 \pm 1.9 \mathrm{ng} / \mathrm{ml})$. sCa was measured by a complexometric method (Corning Glass Works, Corning Science Products, Corning, NY) and in patients with malignant hypercalcemia, serum calcium was corrected for serum albumin (25). sP and sAP were measured by a colorimetric method and uOH-Prol was determined with the method of Kivirikko et al. (26). sPTH was measured with a commercial double-antibody radioimmunoassay (PTH-RIA-100; Cie. ORIS Industrie) that uses a rabbit antiserum directed to bovine PTH with major immunochemical specificity for the carboxy-terminal region of the molecule. cAMP was measured in urine by a solid-phase radioimmunoassay (Immunotech; Luminy, Case 915, Marseille, France). Control values for sCa, sP, sAP, sPTH, uCa, uOH-Prol, and cAMP were obtained in 30 normal adults, 40-60-yr-old.

Bone histomorphometry. A transiliac bone biopsy was performed with a 7.5-mm trephine in 11 patients with primary hyperparathyroidism who gave informed consent. In these patients, the severity of the disease assessed by biochemistry and by bone X-rays did not differ from the general population of 25 patients with primary hyperparathyroidism. In 11 patients with bone metastases with or without hypercalcemia, a transiliac bone biopsy was obtained in a noninvaded area as assessed by $\mathrm{X}$-rays and bone scan after informed consent as part of a prospective study on bone metastases. Bone marrow examination showed metastatic tumoral cells on two biopsies that were excluded in order to rule out the direct local effect of metastases on bone remodeling. We analyzed the following parameters in undecalcified bone biopsy specimens on serial section, $8 \mu \mathrm{m}$ thick, stained with Solochrome Cyanin or by Goldner's process $(27,28)$ : relative resorption surfaces (percent), osteoclast number (per millimeter of trabecular surfaces), relative osteoid volume (percent), relative osteoid surfaces (percent), and the osteoid seam width measured directly according to the method of Kragstrup et al. (29). Histomorphometric data were compared with values obtained in age-matched controls ( 10 women, 5 men) who all had violent deaths.

Statistical analysis. Correlations between biochemical measurements and histomorphometric data were assessed by using linear regression analysis and Spearman's coefficient of rank correlation. Analysis of linear regression was used to determine the relation between the biochemical measurements. Differences between patients and controls were analyzed by the unpaired Student's $t$ test; variations of sBGP values with time were analyzed by the paired Student's $t$ test.

Table I. Clinical Finding in the Nine Patients with Malignant Hypercalcemia

\begin{tabular}{|c|c|c|c|c|c|c|c|}
\hline \multirow[b]{2}{*}{ Patient } & \multirow[b]{2}{*}{ Age $(y r) / \operatorname{sex}$} & \multirow[b]{2}{*}{ Tumor type } & \multirow[b]{2}{*}{$\begin{array}{l}\text { Metastases (other } \\
\text { than bone) }\end{array}$} & \multicolumn{2}{|l|}{ Bone X-ray } & \multirow[b]{2}{*}{$\begin{array}{l}\text { Bone scan } \\
\text { No.‡ }\end{array}$} & \multirow[b]{2}{*}{$\begin{array}{l}\text { Iliac crest } \\
\text { biopsy }\end{array}$} \\
\hline & & & & Type of lesion & No.* & & \\
\hline 1 & 59/Male & Lung & Liver & Lytic & 2 & 2 & Yes \\
\hline 2 & 58/Male & Lung & Liver & Lytic & 3 & 4 & No \\
\hline 3 & 61/Male & Unknown adenocarcinoma & Liver & Lytic & 4 & 8 & No \\
\hline 4 & 47/Male & Lung & Liver (soft tissue) & Lytic & 2 & Not done & No \\
\hline 5 & 39/Male & Testicular dysembryoma & Lung & Mixed & 1 & 1 & No \\
\hline 6 & 60/Male & Lung & Liver (soft tissue) & Lytic & 2 & 4 & Yes \\
\hline 7 & 58/Male & Lung & No & Lytic & 2 & Not done & No \\
\hline 8 & 42/Male & Lung & No & Lytic & 5 & 11 & Yes \\
\hline 9 & 43/Female & Breast & Liver & Mixed & 3 & 9 & Yes \\
\hline
\end{tabular}

* Number of areas of lesions. $¥$ Number of areas of increased uptake. 
Table II. Biochemical Data $( \pm S D)$ in Patients with Primary Hyperparathyroidism and in Patients with Bone Metastases with or without Hypercalcemia

\begin{tabular}{|c|c|c|c|c|}
\hline & \multirow[b]{2}{*}{ Hyperparathyroidism } & \multicolumn{2}{|l|}{ Bone metastases } & \multirow[b]{2}{*}{ Normal } \\
\hline & & With hypercalcemia & Without hypercalcemia & \\
\hline & $n=25$ & $n=9$ & $n=15$ & $n=30-130$ \\
\hline \multicolumn{5}{|l|}{ Blood chemistry } \\
\hline $\mathrm{BGP}(n g / m l)$ & $14.2 \pm 9.6 \S$ & $3.1 \pm 2.6 \S^{11 \pi}$ & $6.6 \pm 2.7$ & $6.2 \pm 2.1$ \\
\hline Calcium $(m g / m l)$ & $11.6 \pm 1.2 \S$ & $12.3 \pm 2.5 \ddagger \S \pi$ & $9.2 \pm 0.4$ & $9.7 \pm 0.3$ \\
\hline Phosphate $(m g / d l)$ & $2.8 \pm 0.4 \S$ & $3.8 \pm 0.5$ & $3.4 \pm 0.3$ & $3.7 \pm 0.5$ \\
\hline Alkaline phosphatase (Bodansky U/liter) & $4.6 \pm 1.8 \S(23)^{*}$ & $13.3 \pm 7.58^{11 \pi}$ & $4.8 \pm 3.1 \S$ & $3.0 \pm 1.5$ \\
\hline PTH $(m I U / m l)$ & $8.4 \pm 9.08$ & $2.8 \pm 0.6(7)$ & $2.8 \pm 0.7$ & $2.8 \pm 0.9$ \\
\hline \multicolumn{5}{|l|}{ Urine chemistry/24-h } \\
\hline Calcium ( $m g$ ) & $366 \pm 147 \S(22)$ & $480 \pm 153 \S \pi$ & $168 \pm 122$ & $207 \pm 35$ \\
\hline Hydroxyproline ( $m g$ ) & $83 \pm 57 \S(23)$ & $126 \pm 46 \S^{11 \pi}$ & $50 \pm 33(14)$ & $38 \pm 16$ \\
\hline cAMP $(\mu \mathrm{mol})$ & $7.84 \pm 5.8(20)$ & 6.8 & - & $3.08 \pm 1.25$ \\
\hline
\end{tabular}

* Number of observations. $\ddagger 12.5 \pm 2.5$ after correction for serum albumin. $§ P<0.05-0.001$ vs. normal. " $P<0.05-0.001$ vs. primary hyperparathyroidism. If $P<0.01-0.001$ vs. bone metastases without hypercalcemia.

\section{Results}

Biochemical data in untreated patients (Tables II and III). Despite similar values of $\mathrm{sCa}, \mathrm{sBGP}$ was significantly increased in patients with primary hyperparathyroidism and significantly decreased in patients with malignant hypercalcemia. Mean sBGP was normal in patients with bone metastases without hypercalcemia (Fig. 1). A similar pattern was observed after logarithmic transformation of sBGP values. As expected, mean sP was decreased and mean values of sPTH, sAP, uCa, and uOH-Prol were increased in patients with primary hyperparathyroidism. In contrast to sBGP that was elevated in 17 of the 25 patients, sAP and uOH-Prol were elevated in 7 and 10 patients, respectively. Seven patients, in whom a parathyroid adenoma was sub-

Table III. Correlations between Biochemical Parameters in Patients with Primary Hyperparathyroidism

\begin{tabular}{llll}
\hline & $n$ & $r$ & $P$ \\
\hline sBGP vs. & & & \\
sPTH & 24 & 0.90 & $<0.001$ \\
sCa & 25 & 0.73 & $<0.001$ \\
Adenoma wt & 14 & 0.79 & $<0.001$ \\
sP & 25 & 0.18 & NS \\
sAP & 24 & 0.17 & NS \\
uCa & 22 & 0.33 & NS \\
uOH-Prol & 23 & 0.30 & NS \\
sPTH vs. & & & \\
sCa & 24 & 0.88 & $<0.001$ \\
sAP vs. & & & NS \\
sCa & 24 & 0.03 & NS \\
sPTH & 24 & 0.02 & $<0.05$ \\
uOH-Prol & 23 & 0.46 & NS \\
uOH-Prol vs. & & & NS \\
sCa & 23 & 0.27 & \\
sPTH & 23 & 0.29 & \\
& & & \\
\hline
\end{tabular}

sequently removed, had normal values of sPTH; three of them had a marked elevation of SBGP, whereas SAP was normal. sBGP was highly correlated with $\mathrm{sCa}, \mathrm{sPTH}$, and the adenoma weight, but not with SAP and uOH-Prol. In contrast to SBGP, sAP and uOH-Prol did not reflect PTH secretion as assessed by $\mathbf{S P T H}$, adenoma weight, and sCa. In patients with malignant hypercalcemia there was a marked increase of SAP because of hepatic metastases in two-thirds of the cases. SPTH was normal or low in all patients. $\mathrm{uCa}$ and $\mathrm{uOH}-\mathrm{Prol}$ were significantly higher in malignant hypercalcemia than in controls and than in bone metastases without hypercalcemia.

Bone histomorphometry (Table IV and V). In patients with primary hyperparathyroidism there was a marked increase of

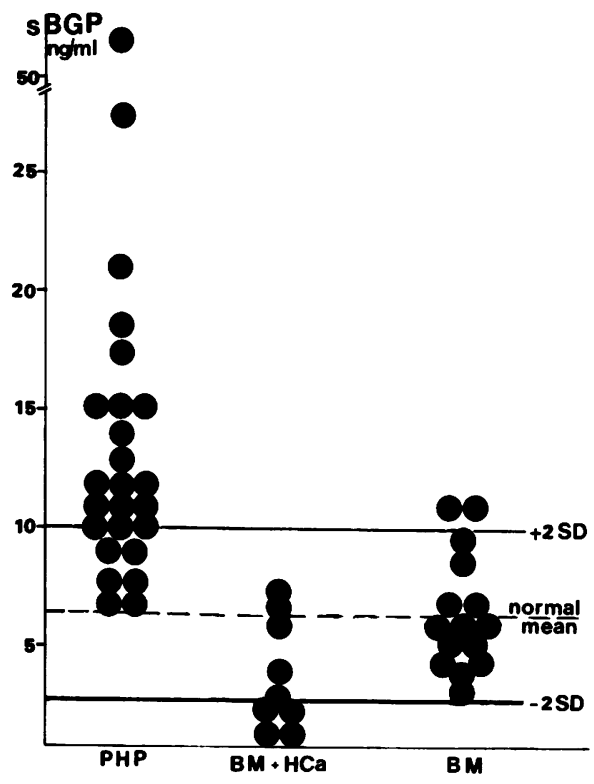

Figure 1. Individual sBGP values in 25 patients with primary hyperparathyroidism (PHP) and in 24 patients having bone metastases with hypercalcemia $(B M+H C a)$ or without hypercalcemia $(B M)$. 
Table IV. Histological Data from Iliac Crest Biopsy in 11 Patients with Primary

Hyperparathyroidism and in 9 Patients with Bone Metastases with or without Hypercalcemia*

\begin{tabular}{|c|c|c|c|c|c|c|}
\hline & Age & $\begin{array}{l}\text { Osteoid } \\
\text { surfaces }\end{array}$ & $\begin{array}{l}\text { Osteoid } \\
\text { volume }\end{array}$ & $\begin{array}{l}\text { Osteoid } \\
\text { thickness }\end{array}$ & $\begin{array}{l}\text { Resorption } \\
\text { surfaces }\end{array}$ & Osteoclasts \\
\hline & & $\%$ & $\%$ & $\mu m$ & $\%$ & $\mathrm{No} . / \mathrm{mm}^{2}$ \\
\hline $\operatorname{Normal}(n=15)$ & $50 \pm 6$ & $11.2 \pm 4.4$ & $1.9 \pm 0.8$ & $10.5 \pm 1.8$ & $3.9 \pm 1.2$ & $0.04 \pm 0.07$ \\
\hline \multicolumn{7}{|c|}{ Primary hyperparathyroidism } \\
\hline$(n=11)$ & $51 \pm 5$ & $31.4 \pm 16 \ddagger \S$ & $6.6 \pm 3.7 \ddagger \S$ & $14.0 \pm 3.5 \S$ & $11.3 \pm 3.3 \ddagger \S$ & $0.48 \pm 0.28 \ddagger$ \\
\hline \multicolumn{7}{|l|}{ Bone metastases with } \\
\hline hypercalcemia $(n=4)$ & $51 \pm 10$ & $14.2 \pm 9.8$ & $1.6 \pm 1.2$ & $5.9 \pm 1.1^{11}$ & $6.4 \pm 4.8$ & $0.34 \pm 0.32 \ddagger$ \\
\hline \multicolumn{7}{|l|}{ Bone metastases without } \\
\hline hypercalcemia $(n=5)$ & $50 \pm 19$ & $20.2 \pm 7.7$ & $3.0 \pm 1.3$ & $8.6 \pm 1.3 \pi$ & $9 \pm 3.7 \ddagger$ & $0.69 \pm 0.40 \ddagger$ \\
\hline
\end{tabular}

* In patients with bone metastases, bone biopsy was performed in a noninvaded area by X-rays and bone scan examination. Histological examination of the marrow did not show any infiltration with malignant cells. $¥$ Higher $(P<0.05-0.001)$ than normal. $\$$ Higher $(P<0.01-0.001)$ than bone metastases with or without hypercalcemia. "Lower than normal $(P<0.001)$ and bone metastases without hypercalcemia $(P<0.05)$.

I Lower than normal $(P<0.05)$.

both osteoclastic resorption and bone formation at the tissue level. In patients with bone metastases, remodeling activity was measured only in nine patients who did not have marrow invasion on the biopsy to exclude the local effect of metastases on bone remodeling. In cancer patients with or without hypercalcemia there was a large increase of bone resorption which contrasted with a decrease of the osteoid seam thickness that was more marked $(t=2.62, P<0.05)$ in patients with malignant hypercalcemia.

Correlation between histological data and biochemical measurements in patients with primary hyperparathyroidism is shown on Table IV; sBGP was significantly correlated with parameters reflecting bone formation but not with those reflecting bone resorption. There was a weak correlation between SAP and osteoid surfaces and between urinary hydroxyproline and resorption surfaces but both correlations did not reach significance. When data from patients having primary hyperparathyroidism and having bone metastases with or without hypercalcemia were pooled (Fig. 2), sBGP was still correlated with the osteoid surfaces ( $r=0.75, n=20, P<0.001)$, the osteoid volume $(r=0.77, n$ $=20, P<0.001)$, and the osteoid seam width $(r=0.77, n$ $=18, P<0.001)$ but not with the resorption surfaces $(r=0.27$, NS) nor with the osteoclasts number $(r=0.31, \mathrm{NS})$.
Effects of treatment of primary hyperparathyroidism on $s B G P$. Repeated biochemical measurements were performed in 16 patients after successful parathyroidectomy. 1 wk after surgery, the drop of sBGP was inconsistent $(14.2 \pm 15.6 \mathrm{ng} / \mathrm{ml}$, vs. $15.7 \pm 11.6 \mathrm{ng} / \mathrm{ml}$ before, $t=0.33$, NS) even after exclusion of the patient showing a dramatic increase of sBGP after parathyroidectomy $(t=1.54, \mathrm{NS})$. At $1 \mathrm{wk}, \mathrm{sBGP}$ was higher than normal $(P<0.05)$, despite normal or low $\mathrm{sCa}$ in all patients $(8.7 \pm 0.16 \mathrm{mg} / \mathrm{dl})$. There was a $47 \%$ decrease of sBGP 2 mo after surgery $(7.2 \pm 3.4 \mathrm{ng} / \mathrm{ml}$ vs. $16.4 \pm 14.3 \mathrm{ng} / \mathrm{ml}, P<0.01)$. sBGP was normalized within 2-6 mo in all patients except one who had high sBGP despite normal sCa and sPTH (Fig. 3). This patient had a moderate renal failure with a serum creatinine of $140 \mu \mathrm{mol} /$ liter that could have influenced sBGP (30).

\section{Discussion}

Deftos et al. (20) reported increased sBGP values in females with primary hyperparathyroidism that were correlated with $\mathrm{sCa}$ and SAP but no data were given on SPTH, adenoma weight, uOH-Prol, and bone histomorphometry. sBGP slightly decreased and remained abnormally high after parathyroidectomy but measurements were done within a month after surgery.

Table V. Correlations between Biochemical Measurements and Histological Data in 11 Patients with Primary Hyperparathyroidism

\begin{tabular}{|c|c|c|c|c|c|}
\hline & Osteoid surfaces & Osteoid volume & Osteoid thickness & Resorption surfaces & Osteoclasts \\
\hline & $\%$ & $\%$ & & $\%$ & $\mathrm{No} . / \mathrm{mm}^{2}$ \\
\hline \multicolumn{6}{|c|}{ sBGP } \\
\hline$r$ & 0.86 & 0.81 & 0.60 & -0.40 & 0.41 \\
\hline$P$ & $<0.001$ & $<0.005$ & NS & NS & NS \\
\hline \multicolumn{6}{|c|}{ Alkaline phosphatase } \\
\hline$r$ & 0.55 & 0.44 & 0.28 & -0.37 & 0.39 \\
\hline$P$ & NS & NS & NS & NS & NS \\
\hline \multicolumn{6}{|c|}{ uOH-Prol } \\
\hline$r$ & 0.13 & 0.03 & -0.63 & 0.52 & 0.26 \\
\hline$P$ & NS & NS & NS & NS & NS \\
\hline
\end{tabular}




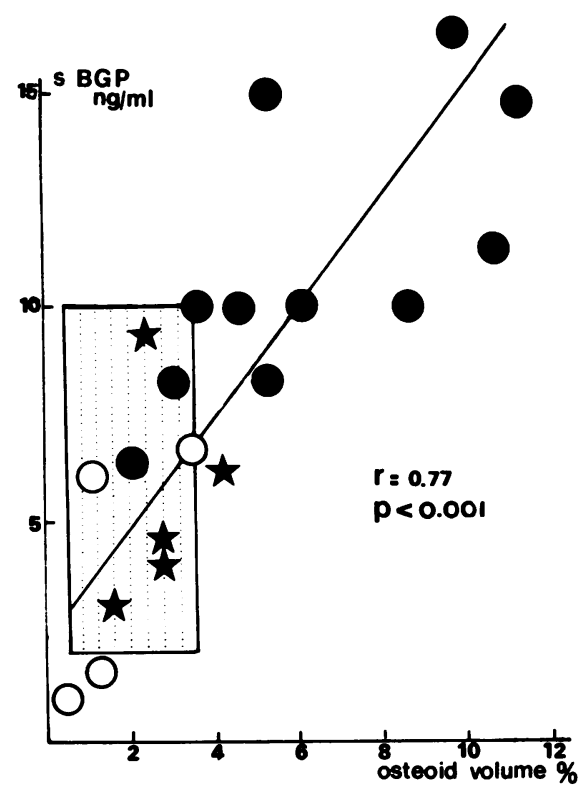

Our finding of increased sBGP values in patients with primary hyperparathyroidism and the highly significant correlation of $\mathrm{sBGP}$ with either $\mathrm{sPTH}, \mathrm{sCa}$, or adenoma weight indicate that $\mathrm{SBGP}$ is a sensitive marker of PTH secretion in this disease. These data are consistent with our previous findings that in normal women, there is an increase in bone turnover with aging with a significant correlation between $\mathrm{SBGP}$ and SPTH (21). The fact that sBGP goes slowly back to normal (within 2-6 mo) after removal of parathyroid adenoma suggests that $\mathrm{SBGP}$ values reflect increased bone turnover at the tissue level rather than a direct effect of PTH on BGP synthesis and/or secretion at the cell level. It is interesting to note that this delay for the normalization of BGP is in agreement with the quantum remodeling theory, as the lifespan of the remodeling activity of a bone structural unit ranges from 4 to $6 \mathrm{mo}(31)$.

An important question is whether sBGP reflects bone formation, bone resorption, or both. Experimental studies in the rat using warfarin (32) and diphosphonates (33) strongly suggest that rat sBGP originates from new cellular synthesis rather than from release from bone matrix during bone resorption. We have previously correlated sBGP with quantitative measurements on iliac crest biopsy in 26 patients with postmenopausal osteoporosis, and we have found that $\mathrm{SBGP}$ is a specific marker of bone formation but does not reflect bone resorption (19). In this study similar findings were observed in patients with primary hyperparathyroidism, indicating that SBGP reflects bone formation

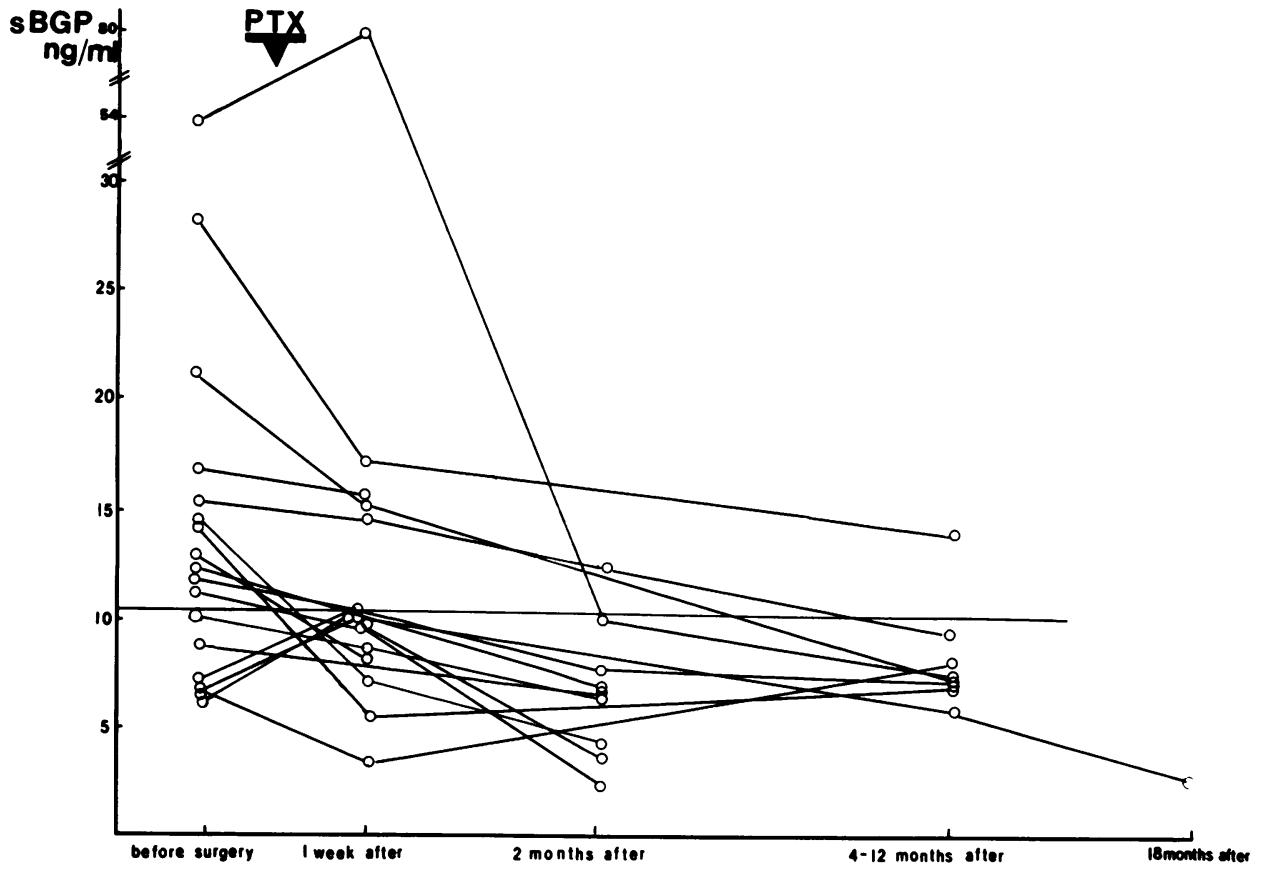

Figure 3. Individual values of $\mathrm{sBGP}$ before and after parathyroidectomy (PTX). 
whether bone turnover is low, normal, or high. BGP released from bone during resorption is probably degraded into small fragments that are not recognized by our antiserum. However, as pointed out earlier, we have no precise information about what controls the fraction of BGP released into the circulation and whether this fraction varies in different disease states. Therefore, no conclusion about the function of BGP can be drawn from this study.

Patients with bone metastases had evidence of increased bone resorption that was higher in patients with hypercalcemia. In contrast to normocalcemic patients who had normal sBGP, hypercalcemic patients had a low SBGP and osteoid seams that were thinner than normocalcemic patients and than controls, both indicating a decreased bone formation. This uncoupling between an increased bone resorption and a decreased osteoblastic activity was probably responsible, at least in part, for the hypercalcemia, but its cause is unknown. Because the bone biopsy was performed in all cases in a nonmetastatic area, this histological pattern cannot be explained by a local effect of tumor cells. As described earlier, it should be noted that the number of bone metastases was not different in patients with or without hypercalcemia. The fact that sBGP was dramatically reduced further supports the hypothesis that an unknown systemic factor might be responsible for the inhibition of bone formation in malignant hypercalcemia. In fact, Stewart has shown that tumoral factors are likely to contribute to hypercalcemia in many patients with metastatic bone disease (7) and it has been recently shown that the degree of hypercalcemia is not related to the extent of metastatic disease detected by bone scans in patients with bone metastases (34). It should be stressed that the same uncoupling between high resorption and low formation has been reported in patients with humoral hypercalcemia of malignancy without any bone metastases (9). Conversely, these patients were in poor health and some degree of immobilization might have influenced, at least in part, the histological pattern. Further studies are required comparing SBGP and bone histomorphometry in a larger sample of patients, including patients with typical humoral hypercalcemia and patients with hypercalcemia related to hematologic disorders. Nevertheless, our data indicate that low sBGP in patients with hypercalcemia and bone metastases reflects a low bone formation.

For clinical purposes, the question is to know if sBGP might be useful for the diagnosis of the etiology of hypercalcemia. In contrast to most PTH assays, sBGP can be measured within a day. Therefore its measurement might be useful for investigating patients with dramatic hypercalcemia, as SBGP will be very high in cases of primary hyperparathyroidism, whereas it will be normal or low in cases of malignant hypercalcemia related to bone metastases. In patients with mild, asymptomatic hypercalcemia due to primary hyperparathyroidism, sBGP was clearly elevated in three patients who had normal sPTH values, suggesting that BGP assay might be useful in such patients when a very sensitive PTH assay is not available.

Bone symptoms, including bone pain and fractures, have become uncommon clinical features in patients with primary hyperparathyroidism $(35,36)$. Evaluating bone turnover with a specific and noninvasive method might be valuable in asymptomatic patients to decide if surgical or medical treatment should be applied and for the follow-up of patients who do not undergo parathyroidectomy. Further studies should be performed to better define the potential benefit of sBGP measurement in the assessment of bone status in primary hyperparathyroidism.

\section{Acknowledgments}

We thank Drs. M. Arlot, E. Saubier (Hotel-Dieu, Lyon), and M. Clavel for their collaboration. The work was supported in part by Cie. ORIS Industrie (contract R098 520 and R098 562).

\section{References}

1. Riggs, B. L., P. J. Kelly, J. Jowsey, and F. R. Keating. 1965. Skeletal alterations in hyperparathyroidism: determination of bone formation, resorption and morphologic changes by microradiography. J. Clin. Endocrinol. Metab. 25:777-782.

2. Byers, P. D., and R. Smith. 1971. Quantitative histology of bone in hyperparathyroidism: its relation to clinical features, X-ray and biochemistry. Q.J. Med. 40:471-480.

3. Meunier, P. J., G. Vignon, J. Bernard, C. Edouard, and P. Courpron. 1973. Quantitative bone histology as applied to the diagnosis of hyperparathyroid states. In Clinical Aspects of Metabolic Bone Diseases. B. Frame, A. M. Parfitt, and H. Duncan, editors. Excerpta Medica, Amsterdam. 215-221.

4. Mosekilde, L., and F. Melsen. 1978. A tetracycline-based histomorphometric evaluation of bone resorption and bone turnover in hyperthyroidism and hyperparathyroidism. Acta Med. Scand. 204:97-106.

5. Parfitt, A. M. 1976. The action of parathyroid hormone on bone. Relation to bone remodeling and turnover, calcium homeostasis and metabolic bone disease. III. PTH and osteoblasts, the relationship between bone turnover and bone loss, and the state of the bones in primary hyperparathyroidism. Metab. Clin. Exp. 25:1033-1069.

6. Charhon, S. A., C. M. Edouard, M. E. Arlot, and P. J. Meunier 1982. Effects of parathyroid hormone on remodeling of iliac trabecular bone packets in patients with primary hyperparathyroidism. Clin. Orthop. Relat. Res. 162:255-263.

7. Stewart, A. F., R. Horst, L. J. Deftos, E. C. Cadman, R. Lang, and A. E. Broadus. 1980. Biochemical evaluation of patients with cancerassociated hypercalcemia. N. Engl. J. Med. 303:1377-1383.

8. Mundy, G. R., K. J. Ibbotson, S. M. D’Souza, E. L. Simpson, J. W. Jacobs, and T. J. Martin. 1984. The hypercalcemia of cancer. $N$. Engl. J. Med. 310:1718-1727.

9. Stewart, A. F., A. Vignery, A. Silvergate, N. D. Ravin, V. Livolsi, A. E. Broadus, and R. Baron. 1982. Quantitative bone histomorphometry in humoral hypercalcemia of malignancy: uncoupling of bone cell activity. J. Clin. Endocrinol. Metab. 55:219-227.

10. Besarb, A., and J. F. Caro. 1978. Mechanisms of hypercalcemia in malignancy. Cancer. 41:2276-2285.

11. Sharp, C. F., R. K. Rude, S. B. Oldham, R. Terry, and F. R. Singer. 1982. Abnormal bone and parathyroid histology in carcinoma patients with pseudo hyperparathyroidism. Cancer (Phil.). 49:1449-1557.

12. McDonnel, G. D., C. R. Dunstan, R. A. Evans, J. N. Carter, E. Hills, S. Y. P. Wong, and D. R. McNeil. 1982. Quantitative bone histology in the hypercalcemia of malignant disease. J. Clin. Endocrinol. Metab. 55:1066-1072.

13. Hauschka, P. V., J. B. Lian, and P. M. Gallop. 1975. Direct identification of the calcium-binding amino acid, $\gamma$ carboxyglutamate, in mineralized tissue. Proc. Natl. Acad. Sci. USA. 72:3925-3929.

14. Price, P. A., A. S. Otsuka, J. W. Poser, J. Kristaponis, and N. Raman. 1976. Characterization of a $\gamma$ carboxyglutamic acid-containing protein from bone. Proc. Natl. Acad. Sci. USA. 73:1447-1451.

15. Nishimoto, S. K., and P. A. Price. 1980. Secretion of the vitamin $\mathrm{K}$ dependent protein of bone by rat osteosarcoma cells. J. Biol. Chem. 255:6579-6583.

16. Price, P. A., and J. K. Nishimoto. 1980. Radioimmunoassay for the vitamin K-dependent protein of bone and its discovery in plasma. Proc. Natl. Acad. Sci. USA. 77:2234-2238.

17. Price, P. A., J. G. Parthemore, and L. J. Deftos. 1980. New biochemical marker for bone metabolism. J. Clin. Invest. 66:878-883.

18. Gundberg, C. M., J. B. Lian, P. M. Gallop, and J. J. Steinberg. 1983. Urinary $\gamma$-carboxyglutamic acid and serum osteocalcin as bone 
markers: studies in osteoporosis and Paget's disease. J. Clin. Endocrinol. Metab. 57:1221-1225.

19. Slovik, D. M., C. M. Gundberg, R. M. Neer, and J. B. Lian. 1984. Clinical evaluation of bone turnover by serum osteocalcin measurements in a hospital setting. J. Clin. Endocrinol. Metab. 59:228-230.

20. Deftos, L. J., J. G. Parthemore, and P. A. Price. 1982. Changes in plasma bone gla-protein during treatment of bone disease. Calcif. Tissue Int. 34:121-124.

21. Delmas, P. D., D. Stenner, H. W. Wahner, K. G. Mann, and B. L. Riggs. 1983. Increase in serum bone $\gamma$-carboxyglutamic acid protein with aging in women. Implications for the mechanism of age-related bone loss. J. Clin. Invest. 71:1316-1321.

22. Delmas, P. D., H. W. Wahner, K. G. Mann, and B. L. Riggs. 1983. Assessment of bone turnover in post menopausal osteoporosis by measurement of serum bone gla-protein. J. Lab. Clin. Med. 102:470476.

23. Brown, J. P., P. D. Delmas, L. Malaval, C. Edouard, M. C. Chapuy, and P. J. Meunier. 1984. Serum bone gla-protein: a specific marker for bone formation in postmenopausal osteoporosis. Lancet. i: 1091-1093.

24. Malluche, H. H., M. C. Faugere, P. Fanti, and P. A. Price. 1984. Plasma levels of bone gla-protein reflect bone formation in patients on chronic maintenance dialysis. Kidney Int. 26:869-874.

25. Payne, R. B., A. J. Little, R. B. Williams, and J. R. Milner. 1973. Interpretation of serum calcium in patients with abnormal serum proteins. Br. Med. J. 4:643-646.

26. Kivirikko, K. I., O. Laitinen, and D. J. Prockop. 1967. Modification of a specific assay for hydroxyproline in urine. Analytical Biochem. 19:249-255.

27. Meunier, P. J., P. Courpron, C. Edouard, J. Bernard, J. P. Bringuier, and G. Vignon. 1973. Physiological senile involution and patho- logical rarefaction of bone. Quantitative and comparative histological data. Clin. Endocrinol. Metab. 2:239-256.

28. Meunier, P. J., J. M. Coindre, C. Edouard, and M. E. Arlot. 1980. Bone histomorphometry in Paget's disease. Quantitative and dynamic analysis of pagetic and nonpagetic bone tissue. Arthritis Rheum. 23:1095-1103.

29. Kragstrup, J., F. Melsen, and L. Mosekilde. 1983. Thickness of lamellae in normal human iliac trabecular bone. Metab. Bone Dis. Rel. Res. 4:291-295.

30. Delmas, P. D., D. M. Wilson, K. G. Mann, and B. L. Riggs. 1983. Effect of renal function on plasma levels of bone gla-protein. $J$. Clin. Endocrinol. Metab. 57:1028-1030.

31. Frost, H. M. 1973. Bone Remodeling and Its Relationship to Metabolic Bone Disease. Charles C. Thomas, Springfield, IL.

32. Price, P. A., M. K. Williamson, and J. W. Lothringer. 1981. Origin of the vitamin $\mathrm{K}$ dependent bone protein found in plasma and its clearance by kidney and bone. J. Biol. Chem. 256:12760-12766.

33. Price, P. A., M. K. Williamson, and S. A. Baukol. 1981. The vitamin $\mathrm{K}$-dependent bone protein and the biological response of bone to 1,25 dihydroxyvitamin D3. In The Chemistry and Biology of Mineralized Connective Tissues. A. Veis, editor. Elsevier/North Holland, Amsterdam. 327-335.

34. Ralston, S. H., I. Fogelman, M. D. Gardiner, and I. T. Boyle. 1984. Relative contribution of humoral and metastatic factors to pathogenesis of hypercalcemia in malignancy. Br. Med. J. 288:1405-1409.

35. Heath, H., S. F. Hodgson, and M. A. Kennedy. 1980. Primary hyperparathyroidism: incidence, morbidity, and potential economic impact in a community. $N$. Engl. J. Med. 302:189-193.

36. Mundy, G. R., D. H. Cove, and R. Fisken. 1980. Primary hyperparathyroidism: changes in the pattern of clinical presentation. Lancet. i: $1317-1320$. 